\title{
Attention, physics and teaching
}

\author{
Lester Ingber \\ Physical Studies Institute, Inc., P.O. Box W; Solana Beach, CA 92075, USA \\ and Institute for Pure and Applied Physical Sciences, University of California San Diego, \\ La Jolla, CA 92093, USA
}

\begin{abstract}
A specific physics problem format has been successful in helping students to develop attentional strategies in solving standard physics problems. The basic structure of this format simultaneously presents alternative patterns of possible solutions/approaches with a correlated package of several related facts/criteria that can be utilized as a foundation to construct a formal analysis of the problem.
\end{abstract}

\begin{abstract}
Attention
Recent research in instructional psychology supports the contention that learning can be enhanced by emphasizing such cognitive processes as attention, motivation, verbal and imaginal encoding, storage and retrieval (Wittrock \& Lumsdaine, 1977). Much of this research is based on other neurological investigations, and recent work has begun to understand neocortical information processing in a biophysics context (Ingber, 1981, in preparation). For example, there has been some success in utilizing instructional/testing strategies for teaching physics that utilize Piaget's model of cognitive development (Barnes, 1977; Fuller, Karplus \& Lawson, 1977). Other similar models appreciate innate and learned abilities to process information along a graded 'depth' dimension, extending from sensory to abstract qualities of information.

However, there also exist innate and learned abilities to process information along a 'breadth' dimension, ranging from a diffused processing of patterned information, to a more focused and selective processing of detailed information (Schneider \& Shiffrin, 1977; Shiffrin \& Schneider, 1977; Zaidel, 1977). For example, it has been my observation that some students, when approaching a physics problem, primarily utilize their focused attention to control analysis to build directly a pattern of information that will hopefully coincide with the solution to a problem. Other students utilize selective attention and analysis as a 'feedback' process, manipulating some preset criteria or agreed-upon formalism that is capable of reducing more complex information to the level of the criteria/formalism. Still other students, in approaching their physics problems, utilize their diffused attention to control a modelling 'feed-forward' process, often 'intuitive', projecting forward patterns of information that approximate the problem but whose solution is more immediately obvious. Many other students approach their problems by alternating or by synchronizing the above two archetypal attention processes in order to pay attention to both the 'forest and the trees'. Most important is the observation that most students exercise a definite propensity to utilize
\end{abstract}


one particular attentional strategy. Learning physics will be discussed, but the following obviously applies quite generally.

A mature student 'naturally' utilizes the appropriate depth and breadth of his/her attentional strategy. It is my contention that many students otherwise capable of applying flexibly and appropriately their depth of attention to physics problems, fail for lack of flexibility in developing strategies to utilize appropriately their breadth of attention. This paper introduces a specific problem format, called the 'ISA Problem' format, which I have designed to help such students, and to aid their teachers to help them develop breadth of attention strategies within the specific context of learning and doing physics. Mature physics students, quite capable of doing their assigned tasks, may also improve their problemsolving abilities, and gain a deeper appreciation for physics and the processes by which physicists approach nature.

\section{Teaching}

A short history of the development of this format illustrates its wide applicability to many levels of physics courses and to many kinds of students:

In 1967, while teaching a graduate course in Advanced Quantum Mechanics on nuclear forces at the level of Ingber (1968) and Ingber \& Potenza (1970) at UC Berkeley, I began attempting to develop teaching methods to help my students become aware of their attentional strategies to work their physics problems. After more attempts at SUNY Stony Brook in 1969-1970 with a graduate course in Methods of Mathematical Physics at the level of Mathews \& Walker (1964), I further developed and employed some formal procedures in an undergraduate course in Natural Science $2 \mathrm{~A}$ at UC San Diego at the level of Halliday \& Resnick (1970). Appendix A presents statistics that support these procedures which were then codified into ISA Problems. In 1972-1973, these ISA Problems worked quite well with a series of six UC San Diego Extension Courses (Ingber, 1972); I taught Application of Karate to the Studies of Attention and Physics at the level of Ingber (1976), and also Developing Intuition for the Solution of Problems in Physics at the level of Feynman, Leighton \& Sands (1964). ISA Problems were also used in a summer session course, Concepts in Physics from 1973-1976 at UCSD at the level of CRM Books (1973).

From 1972 through 1978, I used ISA Problems in a similar course in a small juniorsenior high school (usually 10-16 students, 6-10 part-time staff at the level of graduate student or higher) sponsored by the Institute for the Study of Attention (ISA), a subsidiary of the Physical Studies Institute. Because the ISA Alternative School voted not to have extensive or standardized testing, our perceived successes in developing people are counterbalanced by our lack of statistical evidence to support our contentions. Nevertheless, the positive value, albeit subjective, of our eight years of experience seems worthwhile to report here as support for the ISA Problem format. During this period, all teachers in over 30 academic, fine arts and physical disciplines were trained to create ISA Problems, contributing to a present file of over 2000 problems and complete manuscripts in algebra (S. Bryant \& Ingber, unpublished) and chemistry (Clark \& Ingber, unpublished).

In Winter 1979, I introduced this concept to the UC San Diego music course Holism/ Analysis for performers, composers, visual artists and critics instructed by Pauline Oliveros. Students wrote a weekly ISA Problem on their own concerns. This helped them to share their diverse interests through this common process.

Interestingly, these methods were developed from similar methods I developed during my involvement studying and teaching Karate at Caltech (1958-1962), which were similarly formalized (in 'body language') and taught at UC San Diego (1962-1966; 1970-1972), 
SUNY Stony Brook (1969-1970), and ISA (1970-present) (Ingber, 1976). This text develops karate techniques to be used as sparring tools to study breadth of attention, through a progression of specific exercises. The physical techniques are completely described and organized according to principles of freshman physics: production of forces and torques, linear and angular momentum, expansion-compression of muscular energy. The same methodology and similar exercises were developed for tennis in J. Bryant, Ingber \& Rosi (unpublished). Introductory physics can often best be learned by students if they can concretize the required concepts at a less abstract, sensory level. This addresses similar issues raised Fuller et al. (1977).

However, the most appropriate way to present these ideas here is to illustrate several specific ISA Physics Problems. In fact, prospective ISA teachers were best screened and trained by having them use as source material examples of ISA Problems done by previous teachers in their speciality. Subtleties of composition were discussed after they attempted to create three or four of their own ISA Problems.

\section{ISA Problems}

The following ISA Problems give a student options in intuitive and analytic approaches to problem-solving, and allow him/her to make decisions while being immersed in/supported by mature alternative responses. Presentation of alternative, patterned concepts in the expanded solutions of the left-hand-column (LHC) helps to develop pattern recognition and the use of modelling techniques. Presentation of alternative facts in the right-hand-column (RHC) helps to expose faulty concepts and provides a foundation on which to build an analytic approach. These can vary from simple technical observations to subtle logical distinctions. The set of alternative facts is sufficient to analyse the incorrect solutions, helping students to appreciate and thereby correct their mistakes and weaknesses. Presentation of an intermeshing of both sets of alternatives permits many students to develop a facility to synchronize maximally all this information en route to solving/defining problems. This intermeshing of the two sets of alternatives that span the student's breadth of attention clearly goes beyond presentation of the usual multiple-choice format, which at best requires decision making within a narrow range of the student's breadth of attention dimension.

In each of the following Problems, one solution in the LHC is correct or superior, and the other(s) is(are) incorrect or inferior according to the criteria specified on the RHC. Decide which LHC solution is correct and specify which RHC criterion(-ria) explains why the other LHC solution(s) is(are) incorrect.

In Fig. 1, only the LHC is given.

Now try the solution, given the Related Facts in Fig. 2. The intermeshing (not simple matching, as each solution may have more than one Related Fact) of solutions/patterned approaches with specific facts imparts an experience of understanding the nature and solution to the original problem that many times cannot be duplicated as clearly by a linear exposition.

This ISA Problem also suggests that the nature of the interdependencies of the archetypal attentional processes is also subject and context dependent. This is most easily understood when learning a new subject. For example, to a student just beginning to learn a new language, a sentence may be correlated to a large pattern, and the individual words correlated to an isolated datum. However, to a native speaker, the same sentence may be processed as an isolated datum in the context of a larger paragraph of several sentences. In the above ISA Problem, for instance, a mature student might transcend the collection of separate sentences/calculations to realize that a more general concept, relativity, provides a rationale 
A man (M) and a woman (W), in separate rowboats, are at the same spot in a stream which is moving at the rate of $3 \mathrm{mph}$. $M$ rows at $4 \mathrm{mph}$ upstream (relative to the stream) and $W$ rows at $5 \mathrm{mph}$ downstream for one hour. They then turn $180^{\circ}$ around and row towards each other at each other's previous rate. How long does it take for them to meet from the time they turn around?

\section{First Solution}

In the reference frame of $\mathrm{M}$ or $\mathrm{W}$, the relative speed apart equals the relative speed towards each other. The time is equal to 1 hour.

\section{Second Solution}

Pick a reference frame on the shore. $\mathrm{M}$ moves $1 \mathrm{mph}$ up, $\mathrm{W}$ moves $8 \mathrm{mph}$ down; they move at a relative speed of $9 \mathrm{mph}$.

After they turn, $M$ moves $8 \mathrm{mph}$ down, $\mathrm{W}$ moves $1 \mathrm{mph}$ up; they move at a relative speed of $7 \mathrm{mph}$.

They move apart $9 \mathrm{mph} \times 1$ hour $=$ 9 miles. The time to come together $x$ $7 \mathrm{mph}=9$ miles, or $t=9 / 7$ hour.

\section{Answers}

Fig. 1. LHC of rowing ISA Problem

A man (M) and woman (W), in separate rowboats, are at the same spot in a stream which is moving at the rate of $3 \mathrm{mph}$. $M$ rows at $4 \mathrm{mph}$ upstream (relative to the stream) and $W$ rows at $5 \mathrm{mph}$ downstream for one hour. They then turn $180^{\circ}$ around and row towards each other at each other's previous rate. How long does it take for them to meet from the time they turn around?

\section{First Solution}

In the reference frame of $\mathrm{M}$ or $\mathrm{W}$, the relative speed apart equals the relative speed towards each other. The time is equal to 1 hour.

\section{Second Solution}

Pick a reference frame on the shore. $M$ moves $1 \mathrm{mph}$ up, W moves $8 \mathrm{mph}$ down; they move at a relative speed of $9 \mathrm{mph}$.

After they turn, $M$ moves $8 \mathrm{mph}$ down, W moves $1 \mathrm{mph}$ up; they move at a relative speed of $7 \mathrm{mph}$.

They move apart $9 \mathrm{mph} \times 1$ hour $=$ 9 miles. The time to come together $x$ $7 \mathrm{mph}=9$ miles, or $t=9 / 7$ hour.

\section{Related Facts}

(A) Einstein's principle of special relativity states that the physics of space-time is unchanged when viewed from all reference frames moving at uniform velocities with respect to the observer's frame of reference.

(B) Relative speed between two objects $A$ and $B$ is equal to $V_{A}-V_{B}$, where $V_{\mathrm{A}}$ and $V_{\mathrm{B}}$ are the (vector) velocities of $A$ and $B$, respectively.

(C) If two legs of a right triangle are of lengths 3 and 4 , the hypotenuse has length 5 .

\section{Answers}

The First Solution is correct.

The Second solution is incorrect by Fact B; i.e. $-1-8=-9$, and $8-(-1)=$ $9 \neq 7$. Notice $(-4+\not)-(5+\not z)=-9$ and $(5+\not z)-(-4+\not z)=9$. By Fact $A$, the stream's velocity cancels out. 
for a specific efficient approach. An even more mature student might initially recognize this concept as essentially being the solution to the problem.

As in any teaching situation, when presenting or constructing these problems, differences as well as similarities among students must be considered. Most physics teachers have sufficient maturity to appreciate qualitative differences between the complimentary ranges of breadth of attention required by the LHC and RHC. They can also arrange that the several alternatives within the LHC or the RHC have similar ranges and qualities of information.

Another example in Fig. 3 is concerned with why the sky is blue. This time we start with some isolated data. It can be appreciated that it is a formidable task to construct a coherent model or picture of why the sky is blue. It is very much like putting together a puzzle to form a picture.

The number of separate data presented here, five (six), is usually a strain on many students. The desired result of presenting a Problem should allow students to at least consider either the RHC or LHC alternatives as a package within the maximum span of their breadth of attention. Too many alternatives often results in a collapse of a relatively wide span of attention, and students then often too narrowly focus down to a subset of only one or two of the alternatives. After some minutes, as they become familiar with an appropriate ISA Problem, most students can package both LHC and RHC to span the problem and its import. To best accomplish this, my experience suggests that the number of LHC or RHC alternatives should not exceed four. After a little experience, teachers can develop the proper judgement to decide lower and upper bounds to the total amount of information placed in LHC and RHC that is approximately necessary and sufficient to allow a given group of students to utilize and develop their attentional strategies.

Why is the sky blue? Let $s$ be the average distance between air molecules, and let $d$ be the wave-length of a photon of light.

First Explanation

Answers

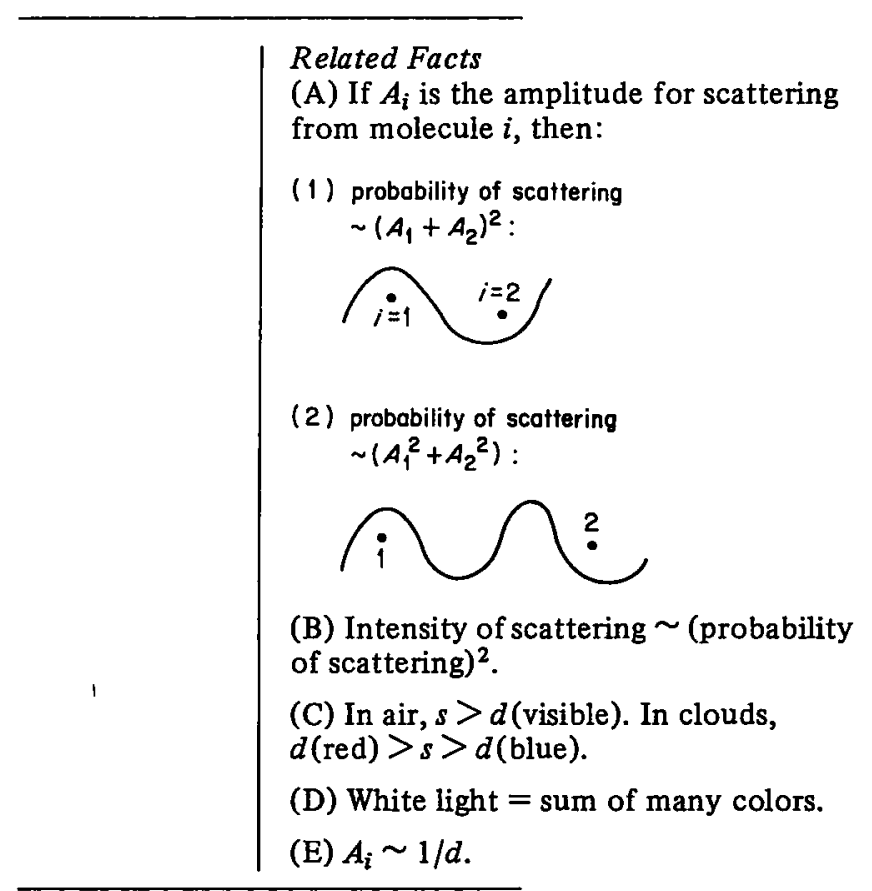


It is an accepted doctrine in physics to respect derived models of interactions as viable knowledge, just as isolated empirical facts or measurements of phenomena are similarly accepted. Having even a couple of models or Explanations at hand to intermesh with the Related Facts in Fig. 4 aids an understanding of the problem. The 'surplus' information in the Problem also suggests some further interesting investigations.

Why is the sky blue? Let $s$ be the average distance between air molecules, and let $d$ be the wave-length of a photon of light.

\section{First Explanation}

The red photons are longer and scatter by $\mathrm{A}-1$. More red is scattered from the sun, leaving it blue.

\section{Second Explanation}

The blue light scatters most since it has the shortest $d$. At an angle away from the sun's main beam, the blue light scatters sufficiently to light the sky blue.

\section{Related Facts}

(A) If $A_{i}$ is the amplitude for scattering from molecule $i$, then:

(1) probability of scottering $\sim\left(A_{1}+A_{2}\right)^{2}$ :

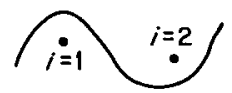

(2) probability of scattering $\sim\left(A_{1}^{2}+A_{2}^{2}\right):$

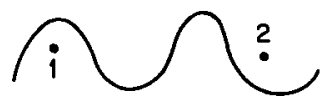

(B) Intensity of scattering $\sim$ (probability of scattering) ${ }^{2}$.

(C) In air, $s>d$ (visible). In clouds, $d$ (red) $>s>d$ (blue).

(D) White light = sum of many colors.

(E) $A_{i} \sim 1 / d$.

\section{Answers}

The Second Explanation is correct.

The First explanation is incorrect by Related Facts B and C, making Fact A-1 irrelevant.

(Extra: Use this information to explain why white clouds and red sunsets appear as they do.)

Fig. 4. RHC and LHC of blue sky ISA Problem

In Fig. 5, much of the clarification to the Problem is left to the answers. Some students might benefit more if this material were given first.

In Fig. 6 graphs are used to reinforce visual patterns while verbal exposition is given to reinforce their analytic content.

As illustrated in Fig. 7, some simple physics ideas have had far-reaching ramifications which tend to expand suddenly our breadth of attention.

In many situations, such as that dealt with in Fig. 8, it can be assumed that the student already knows much of the material, and merely needs to be referred to bodies of knowledge. 
What is the correct relationship between the compression forces $f_{\mathrm{I}}$ and $f_{\mathrm{II}}$, measured between masses $M_{1}$ and $M_{2}$, when the same force $W$ causes the entire system to be accelerated at rate $Y$. Assume $M_{1}>M_{2}$. Use the Related Facts to determine why the other two relationships are incorrect.

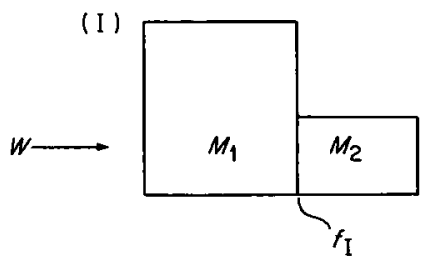

(II)

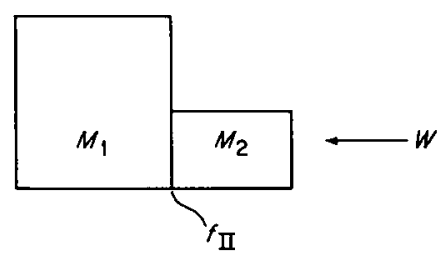

First Relationship

$f_{\mathrm{I}}=f_{\mathrm{II}}$ since the masses receive the same acceleration in each case.

\section{Second Relationship}

$f_{\mathrm{I}}>f_{\mathrm{II}}$ since $M_{1}$ is larger and therefore exerts a greater reaction force on $M_{2}$.

\section{Third Relationship}

$f_{\mathrm{I}}<f_{\mathrm{II}}$ since $M_{2}$ is lighter and therefore more force is required to push $M_{1}$.

\section{Answers}

Let $M_{1}$ be an elephant, $M_{2}$ a peanut, and let $f$ be the force on your finger inserted between $M_{1}$ and $M_{2}$. Clearly, you could have the elephant pushed to push the peanut; but if you have the peanut pushed to push the elephant - ouch!!

Analy tically, $f$ exerts equal and opposite reaction forces on $M_{1}$ and $M_{2}$. Consider case I (Case II is obtained by interchanging $M_{1}$ and $M_{2}$.): $F-f=M_{1} Y, f=M_{2} Y$; or $f=M_{2} Y /\left(M_{1}+M_{2}\right)$. Therefore, $f_{\mathrm{II}}>f_{\mathrm{I}}$, and the Third Relationship is correct.

The First and Second Relationships do not properly take Related Facts A and C into account.

Fig. 5. Elephant and peanut ISA Problem

Which graph most correctly tells us about the forces between the atoms of normal matter, as a function of their relative separation? (Forces at normal matter density $=0$.)

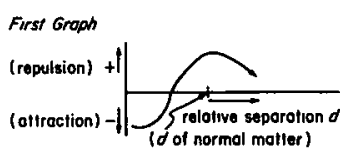

Second Groph
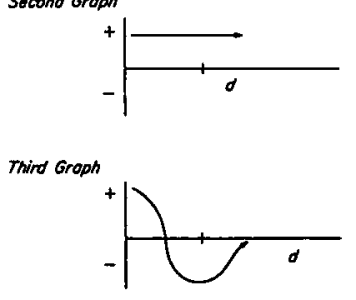

\section{Related Facts}

(A) Place any two atoms a distance apart greater than their separation in normal matter, and they are attracted together.

(B) Place any two atoms a distance apart less than their separation in normal matter, and they are repelled apart.

(C) The pull of any two atoms varies continuously with the distance between them.

(D) Force $=-\Delta$ potential $/ \Delta d$.

Answers

The Third Graph is correct.

The First Graph is incorrect by Related Facts A and B.

The Second graph is incorrect by Related Facts A and B. 
On the basis of the observations (accepted as facts), which theory is most correct?

First Theory

When a force is applied to an object, it is set in motion. When the force is removed, the motion stops.

\section{Second Theory}

Objects in uniform motion tend to stay in uniform motion unless a force is applied.

\section{Observations}

(A) Gravitational force between any two objects is directly proportional to the product of their masses.

(B) If a ball is dropped, it falls straight down.

(C) On any ship at sea going at any uniform speed, the path of a swimming goldfish in a fish bowl on board will be the same.

\section{Answers}

The Second Theory is most correct.

The First Theory (Aristotle's conclusion) is seen to be incorrect by Observation C. By the First Theory a force is necessary to move the ship (and the fish bowl and and the goldfish, etc.). But if the ship is stopped, the motion of the goldfish is the same - the motion is not stopped or altered. Galileo used this argument to refute Aristotle's First Theory on why the earth is at rest. (Can you reproduce such an argument?)

\section{Fig. 7. Aristotle-Galileo motion ISA Problem}

Which of the following describes a reasonable stage of evolution of a star?

\section{First Stage}

As the mass of cosmic gas is contracted, protons and neutrons form deuterium and keep adding neutrons and protons to form the light elements up to and including lithium.

\section{Second Stage}

Two protons interact to form deuterium and provide the fuel to keep the star in stable equilibrium with the gravitational attraction.

\section{Third Stage}

At the extremely high temperatures encountered in the final collapse of a very massive red giant, cool hydrogen from the outer core falls into the center and causes an explosive reaction that blows the star apart.

\section{Related Facts}

(A) A fuel energy of $4 \times 10^{-5} \mathrm{erg}$ is is produced for the overall reaction

(4) hydrogen $1 \rightarrow$ helium 4

(B) Gravitational attraction balances the pressure of thermonuclear reactions in a stable star.

(C) Isotopes of mass 5 are stable for about $10^{-16} \mathrm{~s}$.

\section{Answers}

The Third Stage is correct.

The First Stage is incorrect by Related Fact C.

The Second Stage is incorrect by Related Fact A which gives the relevant reaction.

Fig. 8. Evolution of a star ISA Problem 


\section{Experiences}

A word of caution should put the use of ISA Problems into a proper perspective. My experience has been that these problems are too pedantic to be given 'raw' to most students, without some preliminary introduction such as described below. This is simply because many students are sometimes so concerned/frustrated with the course material, that the appearance of additional structure can be distracting, misleading or even oppressing. These problems are often best used as supplemental material, especially to spark discussion and interaction with the course material.

Also, attempts by some teachers to put too much emphasis on using these ISA Problems for (e.g. weekly) tests did not seem to be as successful as using these problems in a more permissive, flexible, but purposeful environment commensurate with the wide range of breadth of attention to be applied to the specific physics tasks. Most students are initially too anxious/fearful to 'play' with and develop their attentional strategies under testing conditions. Greater emphasis should be placed on 'paying attention' to the alternatives, to working under a time pressure without recorded grades, to getting concrete answers with the best approach, to understanding why an incorrect approach or answer is wrong - in short, to doing physics! As suggested above with regard to the NS 2A class (Appendix A), once these strategies are grasped, albeit in the narrow context of a given class situation, they can apparently be applied to standard problems under standard testing conditions.

A particularly natural way to use these problems is to present them in a fractured fashion, allowing (groups of) students to work/vote on successive approaches towards the completion of the ISA Problems. For example, initially, the several possible LHC solutions may be presented or elicited from the (groups of) students, allowing them to vent/firm up their opinions. Even with the problems presented above, surprisingly, there is usually quite a spread in selections. Then, the several RHC facts/criteria can be presented/elicited, and the students can better appreciate their own strengths and weaknesses. After a few such informal presentations, these ISA Problems can be presented in their entirety to the practiced class. Often, by presenting the same (parts of a) problem on 2 or 3 separate occasions, students who were previously analytically stymied could go on to modify/develop new conceptual approaches. The teacher is left free to roam around the room for 15-30 minutes, to give individualized help according to the specific nature of the part(s) of the ISA Problem with which the student is having difficulty. This is the manner in which I used these ideas in my NS 2A course (Appendix A).

Although some students enjoy composing/solving these problems, another strong feature of this format has been to provide an ongoing teacher-training discipline. By regularly constructing these ISA Problems as an exercise, teachers are continually alerted to be open, flexible and spontaneously creative with alternative ideas and approaches, to elicit precision and completeness, even in casual class situations.

It is obvious that additional demands are placed on the teacher who must create these ISA Problems. This was required of all teachers in the ISA school. However, it seems fair and proper to expect teachers to be at least as creative in the most primitive processes of teaching-interactions, as they expect their students to be in these same processes of learning/ engaging the content of their courses. Perhaps even more important, however, it had been the experience of the ISA school, in having all teachers partake in this ongoing training, that the faculty representing many different disciplines could thereby better discuss the similarities and differences, the strengths and weaknesses, specific and constructive methods, to deal with individual students in their learning and creativity situations. The ISA Problems helped to establish a common language among teachers. We also found it beneficial, sometimes necessary, for teachers to write regularly and present reports orally to the other 
teachers on their specific interactions with a student, analyzed according to the processes and language developed by doing their own ISA Problems. This discipline, besides setting a foundation for meaningful discussion about the students, especially helped those teachers who initially had difficulties in correlating their own intellectual activity of doing ISA Problems with real events transpiring in the classroom.

\section{Conclusions}

My teaching experience supports the contention that people learn in many ways. Some use analysis to methodically build (representations of) pictures and patterns. Others guess, or intuit results, and then, if serious about the problem, they use facts to support and prove their original contentions rigorously. Still others bounce around between analysis and intuition, and when the dust settles, hopefully the problem is solved. Many times the greatest creative satisfaction is obtained by using the last two procedures, because the first method of strict analysis often uses structured formalisms that are already laced with the intuitions of the founders of the subject, but buried after years of tidy analysis, leaving less room to insert one's own intuition. I believe that the purpose of schools should be to provide education in process as well as content, to help people become flexible, creative and adaptive to new situations and demands that are always being made by our ever increasingly complex, technological society. Perhaps by understanding our similarities better, we can better appreciate our differences. Physics may be viewed as a prototypal discipline that offers such an individual and societal service.

\section{References}

Barnes, G. (1977). Scores on a Piaget-type questionnaire versus semester grades for lowerdivision college physics students. Am. J. Phys. 45, 841-847.

CRM Books. (1973). Physical Science Today. Del Mar, CA: CRM Books.

Feynman, R. P., Leighton, R. B. \& Sands, M. (1964). The Feynman Lectures on Physics, Vol. I. Reading, MA: Addison-Wesley.

Fuller, R. G., Karplus, R. \& Lawson, A. E. (1977). Can Physics develop reasoning? Physics Today 30, 23-28.

Halliday, D. \& Resnick, R. (1970). Fundamentals of Physics. New York: Wiley.

Ingber, L. (1968). Nuclear Forces. Phys. Rev. 174, 1250-1263.

Ingber, L. \& Potenza, R. (1970). Nuclear forces and nuclear energetics. Phys. Rev. C 1, 112-122.

Ingber, L. (1972). Editorial: Learning to learn. Explore 7, 5-8.

Ingber, L. (1976). The Karate Instructor's Handbook. Solana Beach, CA: ISA. 96 pp.

Ingber, L. (1981). Towards a unified brain theory. J. social biol. Struct. 4, 211-224.

Mathews, J. \& Walker, R. L. (1970). Mathematical Methods of Physics. 2nd ed. New York: Benjamin. $501 \mathrm{pp}$.

Schneider, W. \& Shiffrin, R. M. (1977). Controlled and automatic human information processing: I. Detection, search, and attention. Psych. Rev. 84, 1-66.

Shiffrin, R. M. \& Schneider, W. (1977). Controlled and automatic human information processing. II. Perceptual learning, automatic attending, and a general theory. Psych. Rev. 84, 127-190.

Wittrock, M. C. \& Lumsdaine, A. A. (1977). Instructional psychology. Ann. Rev. Psychol. $28,417-459$.

Zaidel, E. (1977). Concepts of cerebral dominance in split brain. In (P. Buser \& A. RougeulBuser, Eds.) Cerebral Correlates of Conscious Experience. Amsterdam: Elsevier, pp. 263284. 


\begin{abstract}
Appendix A
Use of ISA Problem format

At UC San Diego I taught a section of 25 students of NS 2A class of 198 students in Winter 1972. I did not help to prepare the standardized weekly, midterm and final exams upon which grades for all students were based. All seven sections had three class lectures and two section recitations per week. The latter included the administration of a weekly class quiz and discussion of material covered in the class lectures and in homework assigned to the class (not graded). Using the bold notation in quotes: 'mean $=n^{-1} \Sigma Y_{i} \equiv \bar{Y} ; n=$ number of students, $Y_{i}=$ a grade on the 4.0 system $/ /$ variance $=(n-1)^{-1} \Sigma\left(Y_{i}-\bar{Y}\right)^{2} / / \mathrm{n}^{\text {': }}$ compared to the class grade of $2.87 / / 1.25 / / 198$, my section achieved $3.44 / / 0.51 / / 25$. The other sections had $2.36 / / 0.97 / / 31,2.52 / / 1.91 / / 23,2.64 / / 2.03 / / 25,2.96 / / 1.25 / / 26,3.04 / / 0.96 / / 25$, $3.09 / / 0.96 / / 43$. I did not teach any of the following quarter's NS $2 B$ class which obtained $2.64 / / 1.37 / / 155$. Students remaining from my NS 2A section were dispersed among the NS 2B sections, and they reverted towards the class norm with $2.81 / / 1.63 / / 16$. (These students had 3.38//0.52//16 in my NS 2A section.) My conjecture is that only one quarter in one subject is insufficient for most students to grasp or to be motivated to develop the strategies described here to the extent they will apply these skills, without prompting or if they are in environments that stress other methods.

In addition to the seven sections of NS 2A, there was also offered a self-paced version NS 2ASP which had the same content and final exam, upon which grades were based. The NS 2ASP class obtained 2.99//1.16//191; the NS 2BSP class obtained 2.84//1.17//2.14. (Students from my NS 2A section with $3.63 / / 0.55 / / 8$ obtained $3.38 / / 1.13 / / 8$ in NS 2BSP, weakly supporting the contention that in the absence of a different structure, students might keep their previous strategies.)

This success prompted ISA to sponsor an alternative school. Courses offered from 19721978 included English (composition/grammar), Creative Writing, Literature, History, Philosophy, Psychology, Economics, Biology, Physical Science (astronomy/chemistry/ physics), Mathematics (algebra/arithmetic/calculus/probability/trigonometry), French, Spanish, Music (instruments/theory), Art (ceramics/drawing/painting), Photography, Dance, Karate, T'ai Chi and Tennis.
\end{abstract}

